\title{
Gut microbial metabolites alter IgA immunity in type 1 diabetes
}

\author{
Juan Huang,,${ }^{1,2}$ James A. Pearson, ${ }^{2}$ Jian Peng, ${ }^{2}$ Youjia Hu, ${ }^{2}$ Sha Sha, ${ }^{2}$ Yanpeng Xing, ${ }^{2}$ Gan Huang, \\ Xia Li, ${ }^{1}$ Fang Hu, ${ }^{1}$ Zhiguo Xie, ${ }^{1}$ Yang Xiao, ${ }^{1}$ Shuoming Luo, ${ }^{1}$ Chen Chao, ${ }^{1}$ F. Susan Wong, ${ }^{3}$ \\ Zhiguang Zhou,' and Li Wen ${ }^{2}$ \\ 'Department of Metabolism and Endocrinology, The Second Xiangya Hospital, Key Laboratory of Diabetes \\ Immunology, Ministry of Education, Central South University, National Clinical Research Center for Metabolic \\ Diseases, Changsha, Hunan, China. ${ }^{2}$ Section of Endocrinology, Department of Internal Medicine, Yale University \\ School of Medicine, New Haven, Connecticut, USA. ${ }^{3}$ Division of Infection and Immunity, School of Medicine, Cardiff \\ University, Cardiff, United Kingdom.
}

The incidence of type 1 diabetes (T1D) has been increasing among children and adolescents, in which environmental factors, including gut microbiota, play an important role. However, the underlying mechanisms are yet to be determined. Here, we show that patients with newly diagnosed T1D displayed not only a distinct profile of gut microbiota associated with decreased short-chain fatty acids (SCFAs) production, but also an altered IgA-mediated immunity compared with healthy control subjects. Using germ-free NOD mice, we demonstrate that gut microbiota from patients with T1D promoted different IgA-mediated immune responses compared with healthy control gut microbiota. Treatment with the SCFA, acetate, reduced gut bacteria-induced IgA response accompanied by decreased severity of insulitis in NOD mice. We believe our study provides new insights into the functional effects of gut microbiota on inducing IgA immune response in T1D, suggesting that SCFAs might be potential therapeutic agents in T1D prevention and/or treatment.

Authorship note: JH and JAP are cofirst authors. LW and ZZ are co-senior authors.

Conflict of interest: The authors have declared that no conflict of interest exists.

Copyright: (c) 2020, American Society for Clinical Investigation.

Submitted: December 20, 2019

Accepted: April 9, 2020

Published: May 21, 2020

Reference information: JCI Insight. 2020;5(10):e135718. https://doi.org/10.1172/jci. insight.135718.

\section{Introduction}

Type 1 diabetes (T1D) is an immune-mediated disease resulting from the destruction of insulin-producing pancreatic $\beta$ cells. Recent evidence suggests the incidence of T1D has been increasing at a rate of up to $4 \%$ per year among children and adolescents (1). This increase in incidence is not attributed to genetics alone, as concordance for T1D between twins is less than $50 \%$ and individuals with newly onset T1D exhibit fewer high-risk HLA haplotypes (2-4). Thus, changes in the environment, including the gut microbiota, have been suggested to modulate the susceptibility to T1D. Studies in both NOD mice and humans have identified microbial composition changes between those that develop T1D and those that do not (5-9). In addition, in humans, altered gut microbial composition in early life and microbial immunogenicity have been linked with islet $\beta$ cell autoimmunity $(10,11)$. Moreover, it has been shown in both NOD mouse model and patients with T1D that bacterial mimics of pancreatic autoantigens can be recognized by autoreactive T cells (12-14). Understanding the functional interactions between the gut microbiota and the immune system in T1D beyond the composition is therefore important.

Short-chain fatty acids (SCFAs) are microbial metabolites produced from the fermentation of dietary material by the intestinal microbiota, and the changes in microbiota composition can influence the production of SCFAs. SCFAs, such as acetate, butyrate and propionate, have been shown to induce Tregs and B cell antibody production $(15,16)$. Interestingly, NOD mice given highly butyrated or acetylated diets were protected from T1D development due to increased frequencies of Tregs and reduced frequencies of autoreactive T cells (17). Furthermore, several bacterial pathways contributing to the synthesis of SCFAs are more prominent in healthy controls than in individuals with T1D (18). In addition, patients with T1D have reduced levels of circulating SCFAs compared with healthy controls (19). Thus, changes in the levels of SCFAs have a profound impact on host immunity, in this case, islet $\beta$ cell autoimmunity.

IgA maintains gut homeostasis with approximately $40 \mathrm{mg} / \mathrm{kg}$ IgA produced each day in the human intestine (20). Microbes and/or ER stress in intestinal epithelial cells can induce IgA immune responses $(21,22)$. In most cases, IgA induced is polyreactive and can bind to many different types of bacteria (23). 
Interestingly, bacteria bound by $\operatorname{IgA}$ are more colitogenic than non-IgA-bound bacteria in inducing colitis (24). To this end, a recent study showed that serum IgA from individuals with T1D has a differential ability to bind specific bacteria compared with that from healthy controls (25). Furthermore, IL-10 production from recirculating intestinal $\operatorname{IgA}^{+} \mathrm{B}$ cells protected mice from developing autoimmune encephalomyelitis (26). Thus, both IgA $\mathrm{A}^{+} \mathrm{B}$ cells and secreted IgA have important roles in mediating gut homeostasis and the susceptibility to autoimmunity. However, the IgA immune response to the altered gut microbiota and their SCFA productions from individuals with T1D has not previously been established.

In this study, we investigated the stool microbial composition and SCFA productions from pediatric donors with T1D and age- and sex-matched healthy controls to determine what functional impact the gut microbiota from these 2 groups has on the IgA immunity. Using germ-free (GF) NOD mice and specific pathogen-free (SPF) NOD mice, we found that microbial SCFAs, particularly acetate, modulated the IgA immune response and suppressed the generation of germinal center B cells. Most importantly, acetate reduced the infiltration of $\mathrm{CD}^{+} \mathrm{T}$ cells in the islets and ameliorated the progression of insulitis, indicating that SCFAs might be potential therapeutic agents in T1D protection and/or treatment.

\section{Results}

Altered composition of gut microbiota is associated with decreased SCFA production and increased IgA-binding in patients with T1D. To explore the crosstalk of gut microbiota, SCFAs, and IgA immune responses in T1D, we first studied the stool microbiota composition from newly diagnosed pediatric donors (the average duration of diabetes was 5 months and the range was 3 days to 12 months) and age- and sex-matched healthy control subjects (Table 1 and Figure 1A). Of the 19 patients, 18 were positive for one or more of the 3 autoantibodies to glutamic acid decarboxylase, insulinoma associated protein 2, and zinc transporter 8 . None of the healthy controls was positive for any of the islet autoantibodies. We found increased $\alpha$ diversity (Figure 1, B and C), with a significant increase in the relative abundances of Ruminococcaceae and Coprococcus (Firmicutes) at the species level in individuals with T1D compared with control subjects (Figure 1, D and E). In contrast, there was a significant reduction in the relative abundances of Roseburia and Megamonas (Firmicutes) at the species level (Figure 1, F and G). To determine whether the stool microbial composition changes influenced the microbial production of SCFAs, we measured stool microbe-derived SCFAs by gas chromatographic mass spectrometer. We found significant reductions in acetate, butyrate, and propionate concentrations in individuals with T1D compared with age- and sex-matched healthy controls (Figure 1, $\mathrm{H}$ and I; and Supplemental Figure 1A; supplemental material available online with this article; https:// doi.org/10.1172/jci.insight.135718DS1), whereas the levels of hexanoic acid, isobutyrate, valerate, and isovalerate were unchanged (Supplemental Figure 1, B-E). Interestingly, acetate was positively correlated with $\beta$ cell function, as indicated by the level of fasting C-peptide (Figure 1J). It was reported that secretory IgA has the capacity to bind bacteria and regulate the composition and function of gut microbiota (23). More recently, differences in IgA binding to bacteria have been linked to both inflammatory bowel disease and T1D $(24,25)$. Thus, we investigated the proportion of IgA-bound bacteria in our study subjects. We detected a higher proportion of IgA-bound bacteria in individuals with T1D compared with healthy control subjects (Figure 2, A and B). Furthermore, the level of these IgA-bound bacteria was negatively correlated with the concentration of stool SCFAs, including acetate, butyrate, and propionate (Figure 2, $\mathrm{C}-\mathrm{E}$ ), and thus we observed a higher proportion of IgA-bound gut bacteria and lower concentrations of 3 SCFAs. Moreover, serum IgA and stool acetate, but not butyrate or propionate, were negatively correlated in patients with T1D (Supplemental Figure 2, A-C). In addition, stool acetate concentration was negatively associated with the abundance of Eubacterium and Hathewayi (Firmicutes), and stool butyrate concentration was negatively correlated to Enterococcaceae (Firmicutes) level (Figure 2, F-H). Taken together, our data support the hypothesis that newly diagnosed individuals with T1D have altered gut microbiota, resulting in reduced concentrations of SCFAs. Interestingly, the altered gut microbiota promoted an increased IgA response to the bacterial targets, and hence there were more IgA-bound gut bacteria in patients with T1D. These results suggest that SCFAs may regulate bacteria-reactive IgA immune responses.

Stool microbiota from individuals with T1D enhance intestinal permeability and alter immune cell function. To directly test the function of the gut microbiota from patients with T1D to stimulate host immune responses in vivo, we orally gavaged GF NOD mice with stool microbiota from 4 randomly selected patients with T1D and their matched healthy controls (Supplemental Table 1 and Figure 3A). We demonstrated that the gut bacterial composition of the mice that received fecal bacteria from individuals with T1D showed clear 
Table 1. Characteristics of patients with newly diagnosed type 1 diabetes and age- and sex-matched control individuals

\begin{tabular}{ccc}
\hline & Type 1 diabetes & Healthy controls \\
$n$ (females/males) & $19(12 / 7)$ & $19(12 / 7)$ \\
Age (yr) & $9.2(8.0-12.0)$ & $10.3(6.5-11.5)$ \\
Fasting glucose (mmol/L) & $6.7(5.2-9.3)^{A}$ & $4.8(4.3-5.1)$ \\
HbA1c (\%) & $9.3 \pm 2.8$ & N/A \\
Fasting C-peptide (pmol/L) & $94.0(34.0-137.3)$ & N/A \\
1-h postprandial C-peptide (pmol/L) & $156.7(53.5-299.1)$ & N/A \\
2-h postprandial C-peptide (pmol/L) & $218.9(83.6-329.9)$ & N/A \\
T1A/T1B & $18 / 1$ & N/A
\end{tabular}

Data are presented as mean \pm SEM or median (25th-75th percentile) as appropriate; N/A, not available. ${ }^{A} P<0.001$. Data were assessed for significance using a 2-tailed Student's $t$ test.

separation by donor (Figure 3B). This contrasted with that from the mice that received fecal bacteria from control donors, in which the gut bacterial composition clustered together (blue circles in Figure 3B). Our results also revealed significant differences at the species level between the 2 groups (Figure 3C). To determine bacterial effects on the gut barrier in vivo, we assessed gut permeability and intestinal antimicrobial peptide expression. Mice that received fecal bacteria from T1D donors exhibited increased gut permeability in vivo (Figure 3D). Of 7 intestinal antimicrobial peptide genes tested, cathelicidin-related antimicrobial peptide (Cramp) was downregulated in the mice that received fecal bacteria from the patients (Figure $3 \mathrm{E}$ and Supplemental Figure 3, A-F). This suggests that altered bacterial composition from the donors with T1D predominantly changed gut permeability and more selectively altered the antimicrobial peptide responses in the intestine. Given that we found a significantly altered IgA immune response to the fecal bacteria in individuals with T1D (Figure 2B), we next assessed IgA immunity in GF NOD mice colonized with these bacteria. Compared with the controls, we found that the mice that received fecal bacteria from individuals with T1D had decreased percentages of $\operatorname{IgA}^{+} \mathrm{B}$ cells in the pancreas-draining lymph node (PLN) (Figure $3 \mathrm{~F}$ ) and increased proportions of IgA-bound bacteria in the cecum, colon, and stool (Figure 3, G and $\mathrm{H}$ ), the latter of which are consistent with our human data (Figure 2B). Furthermore, we found reduced free IgA antibodies in the colon and stool of the mice colonized with fecal bacteria from patients with T1D compared with the control subjects (Supplemental Figure 4A). Interestingly, the GF recipients colonized with fecal bacteria from patients with T1D had increased frequencies of transitional B cells $\left(\mathrm{CD} 21^{-} \mathrm{CD} 23^{+}\right)$ and reduced frequencies of marginal zone $\mathrm{B}$ cells $\left(\mathrm{CD} 21^{+} \mathrm{CD} 23^{-}\right)$in PLN and mesenteric lymph node (MLN) (Supplemental Figure 4, B-C). The bacteria from patients with T1D were also less able to induce Tregs in the large intestinal lamina propria compared with the bacteria from control subjects (Supplemental Figure 4D). In addition, we identified increased frequencies of $\mathrm{CD} 4^{+} \mathrm{IFN}-\gamma^{+}$and $\mathrm{CD} 8^{+} \mathrm{TNF}-\alpha^{+} \mathrm{T}$ cells in the mice colonized with fecal bacteria from patients with T1D compared with the mice that received bacteria from control donors (Supplemental Figure 4, E and F). To determine whether the immune cell function was affected by exposing to gut bacteria from patients with T1D, we stimulated the splenocytes from GF mice colonized with gut bacteria from either patients with T1D or healthy control subjects with anti-CD40 or anti-CD3 mAb. We found that the GF recipients colonized with the bacteria from patients with T1D had increased proliferative responses of immune cells to anti-CD40 and anti-CD3 stimulation compared with GF recipients colonized with bacteria from healthy control subjects (Supplemental Figure 5, A and B). We also stimulated the splenocytes with microbial products - LPS, CpG, and Pam3Csk (Supplemental Figure 5, C-E). Interestingly, there were no significant differences when splenocytes were stimulated with LPS or CpG; however, Pam3Csk stimulation induced higher proliferative responses in splenocytes from GF recipients colonized with gut bacteria from patients with T1D compared with GF recipients that received bacteria from healthy control subjects (Supplemental Figure 5, C-E). Taken together, our results suggest that gut bacteria from individuals with T1D promote a "leaky" gut, alter B cell IgA immune responses, and induce proinflammatory $\mathrm{T}$ cell responses.

SCFAs modulate gut microbiota-mediated IgA immune response. SCFAs are important for maintaining the intestinal barrier and promoting immune homeostasis (16). We have demonstrated, when tested directly ex vivo, that individuals with T1D had altered IgA immune responses compared with healthy control subjects, 
A

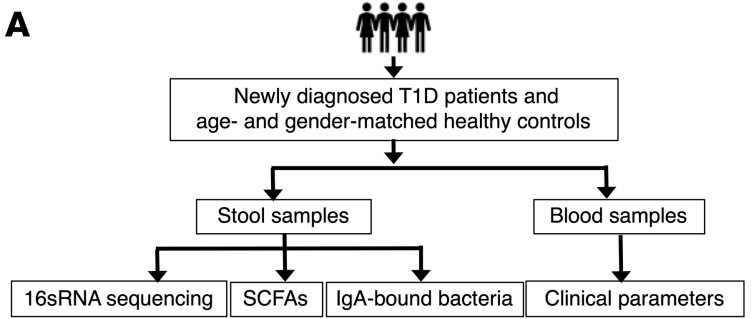

B

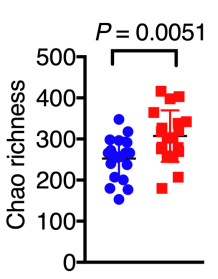

C

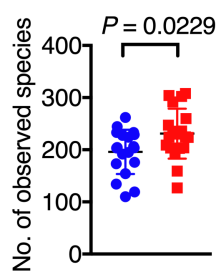

F

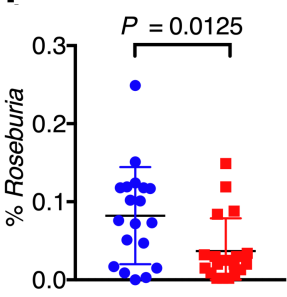

G

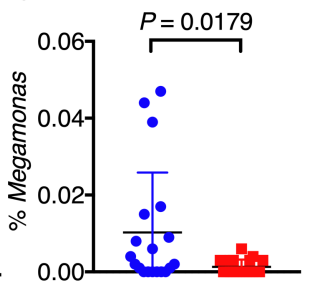

H
I

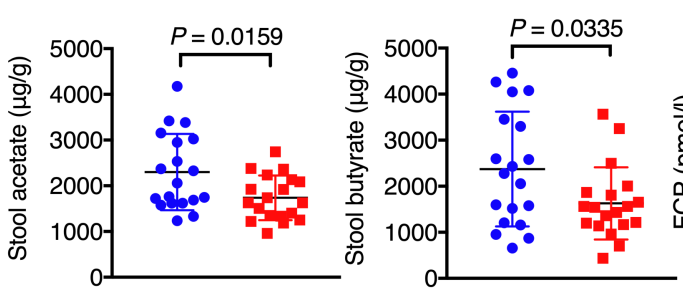

- T1D patients
D

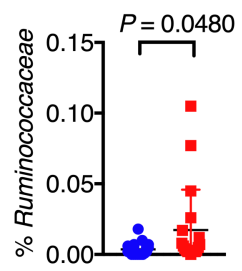

E

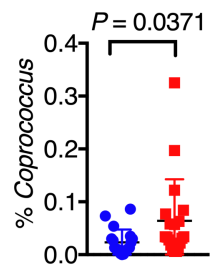

J

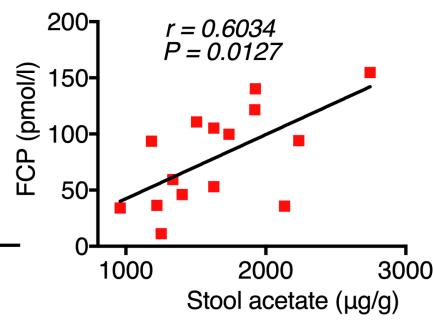

Figure 1. Stool SCFA production and bacteria-targeting IgA response in individuals with T1D compared with control subjects. (A) Experimental design of the study in patients with T1D and healthy control subjects. (B-C) Stool microbiota composition was investigated by $165 \mathrm{rRNA}$ sequencing ( $n=19 / \mathrm{group}$ ). Changes in $\alpha$ diversity were assessed by Chao richness (B) and number of observed species (C). Altered relative microbial abundances of Ruminococcaceae (D), Coprococcus $(\mathbf{E})$, Roseburia $(\mathbf{F})$, and Megamonas $(\mathbf{C})$ between donors with T1D and controls are shown. (H and I) Stool acetate $(\mathbf{H})$, and butyrate (I) concentrations from individuals with T1D and control subjects ( $n=19 /$ group). (J) Correlation between stool acetate level and serum fasting C-peptide concentration $(n=15)$. Data are presented as mean \pm SEM and were analyzed by a 2-tailed Student's $t$ test $(B-I)$. Data in $(J)$ was analyzed using a 2-tailed Pearson correlation coefficient test and linear regression. SCFAs, short-chain fatty acids; T1D, type 1 diabetes.

similar results were also found in GF mice when colonized with the gut bacteria from patients with T1D. Therefore, we hypothesized that the altered IgA response was mediated through the changes in microbially derived SCFAs. To determine the relationship of the IgA response with SCFAs in T1D, we cultured the splenocytes from SPF NOD mice with SCFAs (acetate, butyrate or propionate), in the presence of LPS and anti-CD40 mAb. Interestingly, all tested SCFAs inhibited the IgA production (Figure 4A). Given the negative correlation between the acetate concentration in stool samples and the serum IgA level in patients with T1D (Supplemental Figure 2A), we focused on investigating the effect of acetate on IgA immune responses in vivo. To this end, we colonized GF NOD mice with stool bacteria from T1D donors, followed by gavaging the mice with either acetate or sterile water $\left(\mathrm{H}_{2} \mathrm{O}\right.$, solvent for acetate) (Figure $\left.4 \mathrm{~B}\right)$. It is intriguing that acetate treatment for only 1 week significantly altered the $\alpha$ diversity of gut microbiota (indicated by Chao richness, Figure 4C) and the relative abundance of Porphyromonadaceae (Bacteroidetes) and Staphylococcus (Firmicutes) at the family level in the stools of the formerly GF NOD mice (Figure 4, D and E). In addition, acetate decreased $\mathrm{CD}^{+} \mathrm{T}$ cell proportion in the Peyer's patch (PP) (Figure 4F) and increased the proportion of Tregs (Figure $4 \mathrm{G}$ ). IgA $\mathrm{Ig}^{+} \mathrm{B}$ cells were significantly reduced in the PLN (Figure $4 \mathrm{H}$ ). $\operatorname{IgA} \mathrm{A}^{+}$ $\mathrm{B}$ cells, as well as $\mathrm{IgM}^{+} \mathrm{B}$ cells, were also reduced in the bone marrow of acetate-treated mice (Figure $4 \mathrm{I}$ ). Moreover, free IgA content in the cecum was reduced in acetate-treated mice (Figure 4J). These data suggest that microbial metabolite SCFAs regulate IgA immune responses in NOD mice.

Long-term treatment with acetate reduces IgA binding to bacteria, inhibits germinal center $B$ cell formation, and ameliorates insulitis. To investigate the long-term effects of acetate on modulating IgA immune responses and $\beta$ cell autoimmunity before T1D development in vivo in a conventional setting, we treated SPF NOD mice with acetate for 10-12 weeks (Figure 5A). Acetate treatment led to consistent reduction of IgA-bound gut bacteria (Figure $5 \mathrm{~B}$ ) and improved intestinal barrier function, indicated by decreased gut permeability (Figure 5C). Similar to the short-term acetate treatment of formerly GF mice (Figure 4J), long-term treatment of SPF mice also decreased IgA content in the cecal lumen (Figure 5D) and reduced the proportion of IgA-bound bacteria in the small intestine (Figure 5E), which coincided with lower expression of polymeric Ig receptor (Pigr) (Figure 5F). To assess the IgA binding ability to microbes, we studied the bacterial binding ability of $\operatorname{IgA}$, in the intestinal washing or serum from acetate-treated and control mice, to gut bacteria from Rag-deficient NOD mice (devoid of antibodies). Acetate treatment reduced intestinal IgA binding to gut bacteria (Figure 5G), but not serum IgA 
A

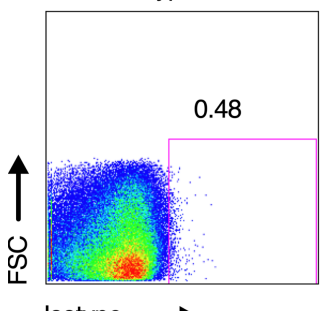

Isotype $\longrightarrow$

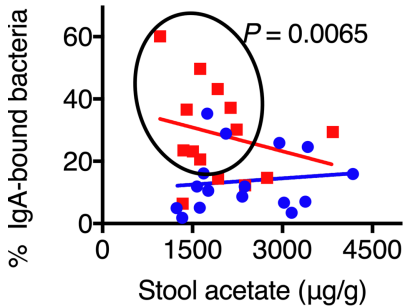

Healthy control

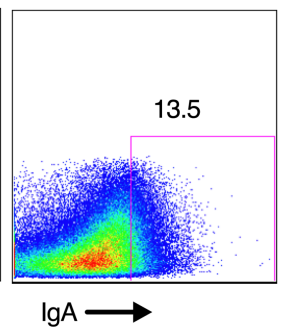

T1D patient

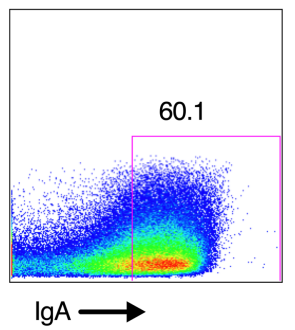

D

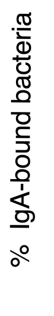

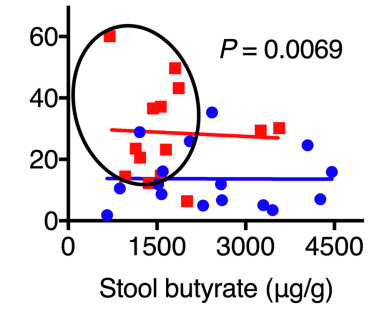

G

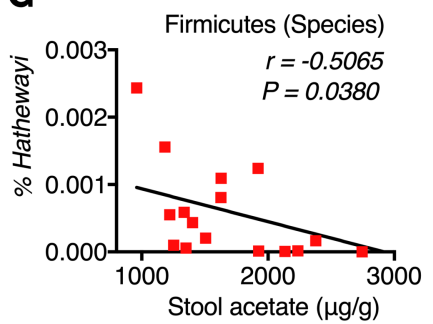

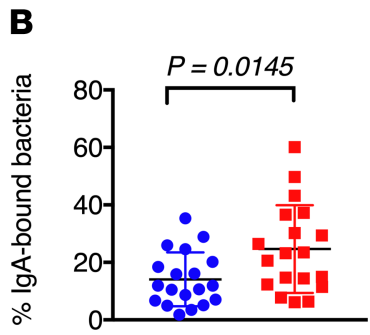

$\mathbf{E}$

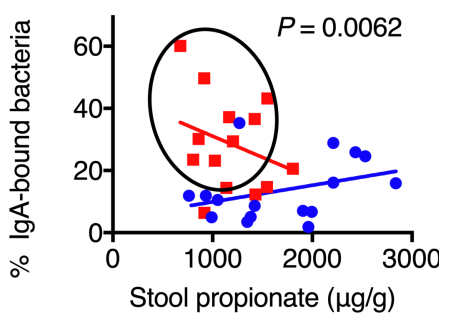

H

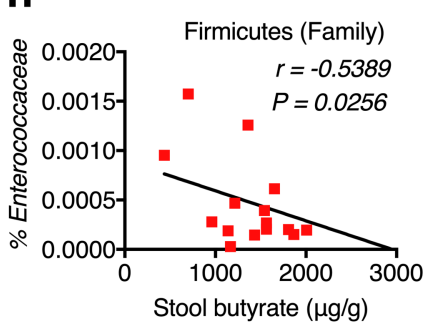

- Healthy controls

T1D patients

Figure 2. IgA-bound bacteria and the correlations with different SCFAs in individuals with T1D and control subjects. (A) Representative flow cytometric profiles of IgA-bound bacteria. (B) Summary of IgA-bound bacteria percentage from donors with T1D and healthy controls ( $n=19$ /group). (C-E) Correlations between stool acetate (C), butyrate (D), or propionate (E) concentration and the level of IgA-bound bacteria. (The overall elevation or intercepts between the 2 groups was compared. The black circles show that there were more patients in the upper left areas, i.e., with a higher percentage of IgA-bound bacteria but lower stool SCFAs, $n=14-16$.) $(\mathbf{F}-\mathbf{H})$ Correlations between stool acetate concentration and the relative abundances of Eubacterium (F) and Hathewayi (Firmicutes) (G), and between stool butyrate concentration and Enterococcaceae (Firmicutes) abundance (H) ( $n=17 /$ group). Data are presented as mean \pm SEM and were assessed for statistical significance using a 2-tailed Student's $t$ test (B). Data in (F-H) were analyzed using a 2-tailed Pearson correlation coefficient test and/or linear regression. SCFAs, short-chain fatty acids; T1D, type 1 diabetes.

binding to gut microbiota (data not shown). Acetate administration also reduced both $\mathrm{CD} 19^{+} \mathrm{B}$ cells and $\mathrm{CD} 4^{+} \mathrm{T}$ cells in the PP (Figure $5 \mathrm{H}$ ), whereas the proportion of IgA $\mathrm{A}^{+} \mathrm{B}$ cells was reduced only in the spleen (Figure 5I). However, we observed decreased germinal center (GC) B cells $\left(\mathrm{PNA}^{+} \mathrm{GL} 7^{+}\right.$) in the MLN and PP of acetate-treated mice (Figure 6A), together with reduced $\operatorname{IgA}^{+}$GC B cells in the spleen of mice that received long-term acetate treatment (Figure $6 \mathrm{~B}$ ). We also found reduced $\operatorname{IgA}^{+} \mathrm{B}$ cells in the bone marrow (Figure 6C), which is in line with short-term acetate treatment in formerly GF NOD mice (Figure 4I). Long-term acetate treatment also reduced $\operatorname{IgD}^{+} \mathrm{B}$ cells in the bone marrow (Figure 6C). Importantly, acetate-treated mice displayed ameliorated insulitis, in contrast to the controls (Figure $6, \mathrm{D}$ and $\mathrm{E}$ ), accompanied by reduced $\mathrm{CD} 4^{+} \mathrm{T}$ cell infiltration in the islets (Figure $6 \mathrm{~F}$ ). Our data suggest that acetate reduces IgA immune responses to bacteria and ameliorates $\beta$ cell autoimmunity.

Finally, to investigate the direct effect of acetate on IgA immune responses, we stimulated the purified splenic B cells from acetate-treated or control mice with LPS and anti-CD40 mAb, in the presence of acetate. We found that B cells from acetate-treated mice secreted significantly less IgA compared with those from control mice in vitro (Figure 7A). IL-6, essential for B cell differentiation, maturation, and survival $(27,28)$, was reduced at the cellular level (Figure 7, B and C) and at the secreted molecular level (Figure 7D) when acetate was present. Quantitative PCR (qPCR) analysis of purified splenic 
A
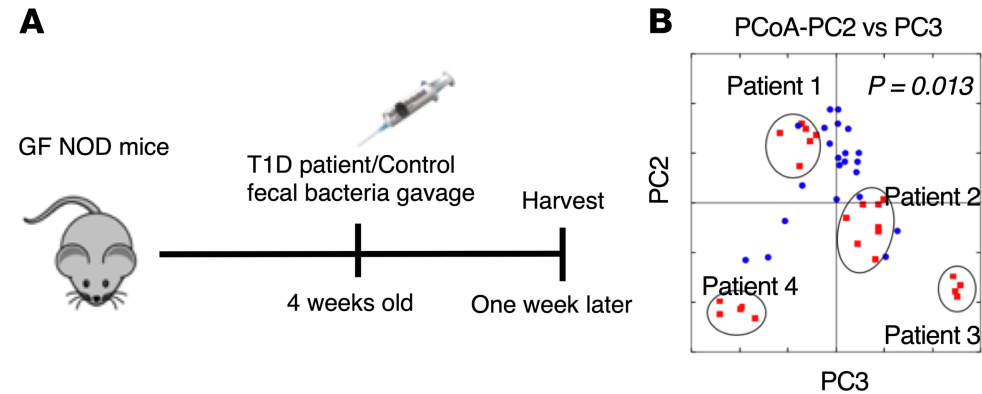

C

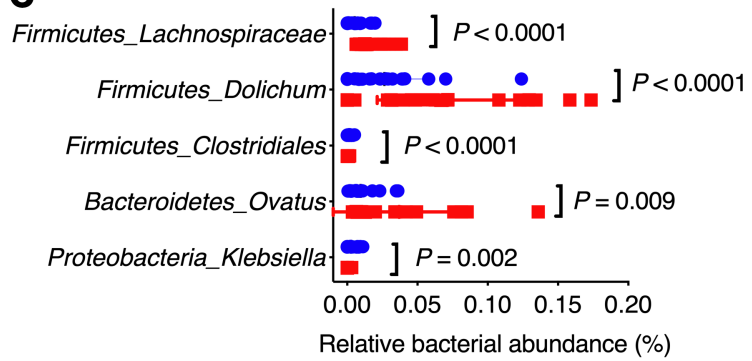

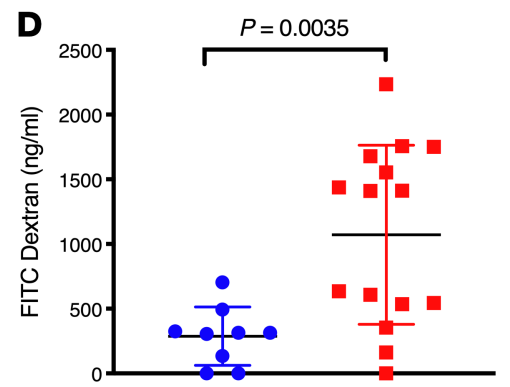
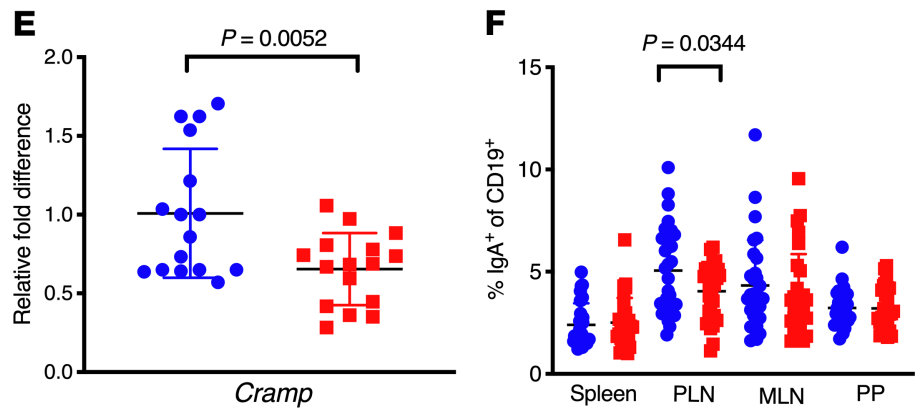

G

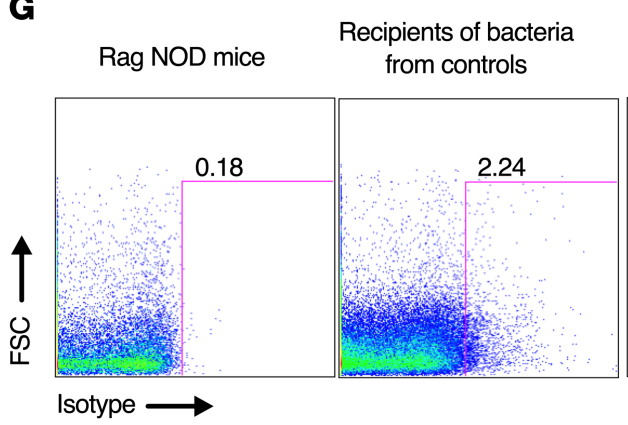

Recipients of bacteria from T1D donors

- Recipients with bacteria from controls

- Recipients with bacteria from donors with T1D

Figure 3. Role of gut microbiota from patients with T1D and control subjects in modulating host immune responses in GF NOD mice. (A) Timeline for GF NOD mice gavaged with stool bacteria from patients with T1D or control subjects. (B) Unweighted principal coordinate analysis of stool microbiota from GF NOD mice received bacteria from either T1D donors or healthy control subjects $(n=22-23)$. (C) Gut microbiota composition at the species level $(n=22-23)$. (D) Gut permeability $(n=9-15)$. (E) Expression of Cramp ( $n=16 /$ group). (F) IgA+ B cell frequency in the spleen, PLN, MLN, and PP $(n=27-30)$. ( $\mathbf{G}$ and $\mathbf{H})$ Representative flow cytometric profiles of IgA-bound bacteria (G), and summary of IgA-bound bacteria percentage (H) Statistical analysis was performed by an analysis of similarities (B), multiple $t$ tests with Bonferroni's correction (C) or a 2-tailed Student's $t$ test (D-F and $\mathbf{H}$, data combined from 2 or more independent experiments are presented as mean \pm SEM). T1D, type 1 diabetes; GF, germ-free; PLN, pancreatic lymph node; MLN, mesenteric lymph node; PP, Peyer's patch.

B cells from acetate-treated mice demonstrated reduced expression of postswitch transcripts $\alpha(P s t \alpha)$, Pst2 $\beta$, Stat $5 b$, and interferon regulatory factor 4 (Irf4) compared with those from control mice (Figure 7, E-H), all of which are important for B cell differentiation. However, there were no significant differences in the expression of activation-induced cytidine deaminase (Aid), X-box-binding protein 1 (Xbp1), paired box 5 (Pax5), and pr-domain zinc finger protein 1 (Prdm1) (Supplemental Figure 6, A-D). Although SCFAs were reported to induce intracellular signaling changes by activating the orphan metabolite-sensing G-protein receptors (GPR41) and GPR43 (29), we did not find any changes in Gpr41 or Gpr43 expression between acetate-treated and nonacetate-treated donor B cells (Figure 8, A and B). However, a member (A7) of solute carrier family 16 (SLC16) transporting SCFAs (30) was significantly reduced on $B$ cells from acetate-treated mice (Figure $8 \mathrm{C}$ ), whereas the other members that were tested were not affected (Figure 8, D and E). Taken together, our findings suggest that acetate treatment alters bacteria-targeting IgA immune responses and affects B cells by reducing IL-6 production and GC B cell generation, most likely mediated through SLC16A7 pathway. 


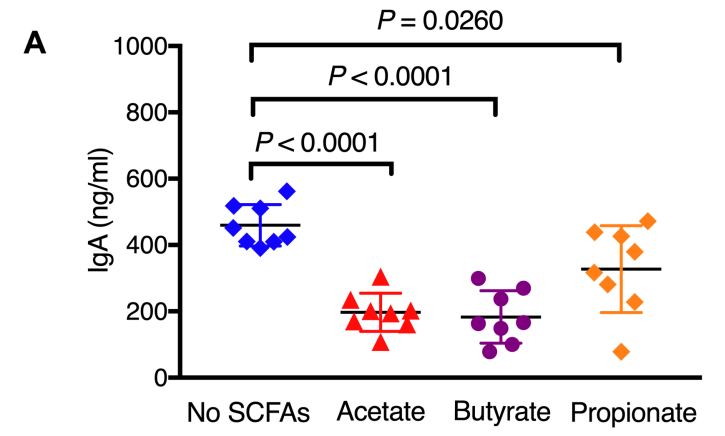

B
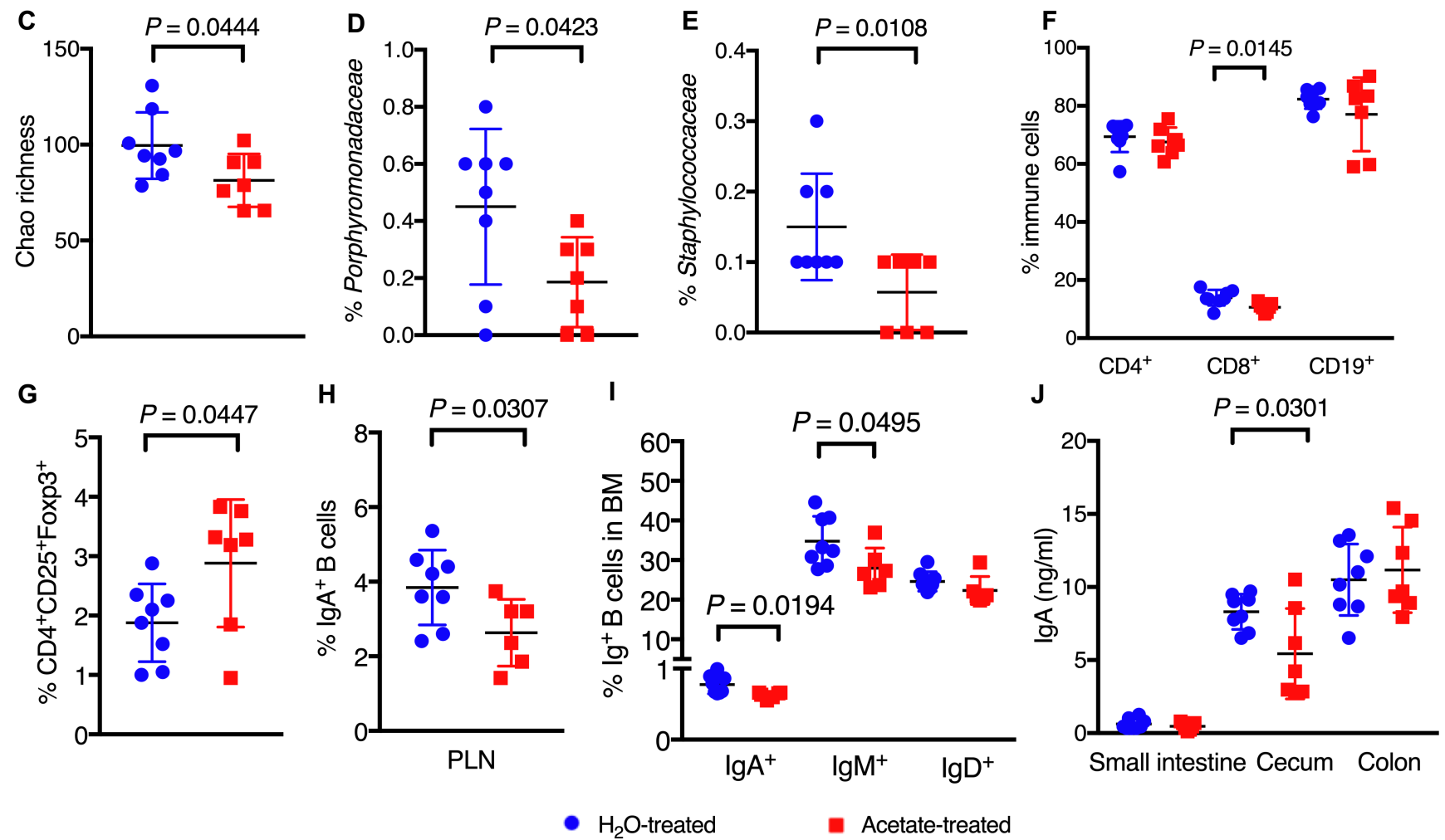

Figure 4. Role of acetate in modulating gut microbiota composition and IgA immune response. (A) Spleen cells from specific pathogen-free NOD mice were stimulated with acetate, butyrate, or propionate (all $0.1 \mathrm{Mm})$ in the presence of anti-CD40 mAb $(20 \mu \mathrm{g} / \mathrm{mL})$ and LPS (10 $\mu \mathrm{g} / \mathrm{mL})$, and secreted IgA in the culture supernatant was measured ( $n=8 /$ group). (B) Timeline of GF NOD mice gavaged with stool bacteria from patients with T1D, followed by acetate or water gavage. (C-E) Gut microbiota in fecal samples were analyzed by 165 rRNA sequencing $(n=7-8)$. Chao richness (C), and relative abundance of Porphyromonadaceae (D) and Staphylococcaceae (E) at family level. (F) Proportion of TCR $\beta^{+}$CD4 ${ }^{+}$T cells, TCR $\beta^{+}$CD $8^{+}$T cells, and TCR $\beta$-CD19+ B cells in PP $(n=7-8)$. (C) Frequency of CD4+CD25+Foxp3+ Treg cells in Peyer's patch $(n=7-8)$. (H) Proportion of IgA ${ }^{+}$B cells in PLN $(n=6-8)$. (I) Proportion of $\mathrm{IgA}^{+}$, IgM+, and IgD+ B cells in bone marrow $(n=7-8)$. (J) IgA concentration from the content of small intestine, cecum and colon $(n=7-8)$. Data combined from 2 independent experiments are presented as mean \pm SEM and were analyzed with a 1-way ANOVA, followed by a Tukey's test with Dunn's correction for subsequent multiple comparisons between 2 groups (A) or a 2-tailed Student's $t$ test (C-J). GF, germ-free; T1D, type 1 diabetes; SCFAs, short-chain fatty acids; PLN, pancreatic lymph node.

\section{Discussion}

In this study, we report what we believe are several novel findings: (a) newly diagnosed patients with T1D show increased proportion of stool IgA-bound gut bacteria, which is negatively associated with the concentrations of some SCFAs, especially acetate; (b) level of stool acetate is negatively associated with circulating IgA concentration; (c) colonization of GF NOD mice with stool bacteria from patients with T1D or healthy control subjects revealed distinct profiles of gut microbiota in the colonized GF NOD mice; (d) gut microbiota from patients with T1D enhanced gut permeability; (e) similar to the stool samples seen in the patients with T1D, GF NOD mice colonized with stool bacteria from the 
A

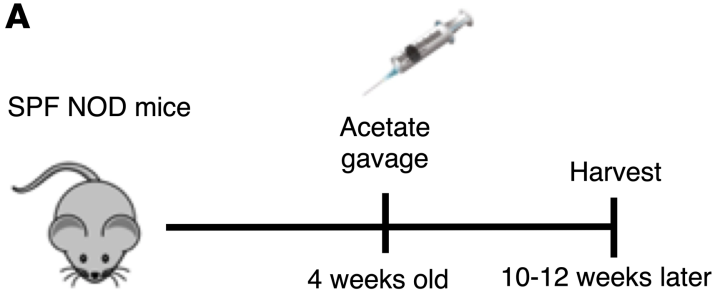

B

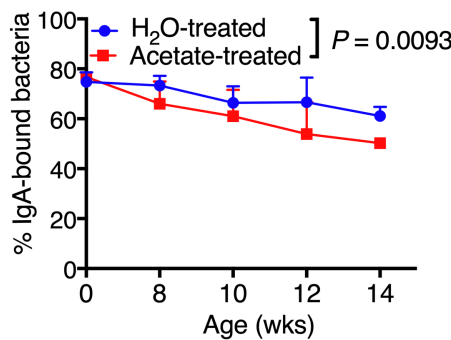

C

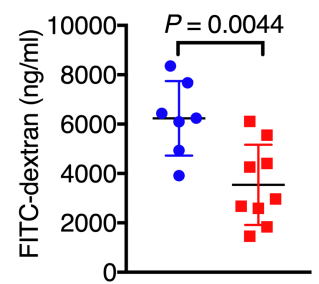

D

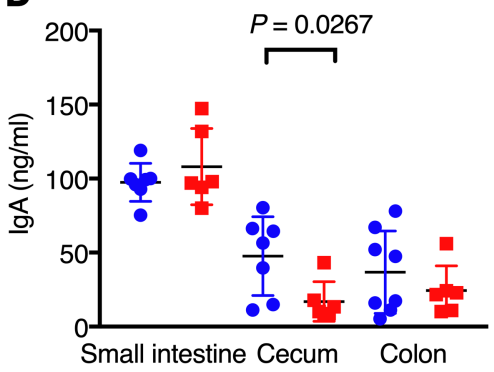

E

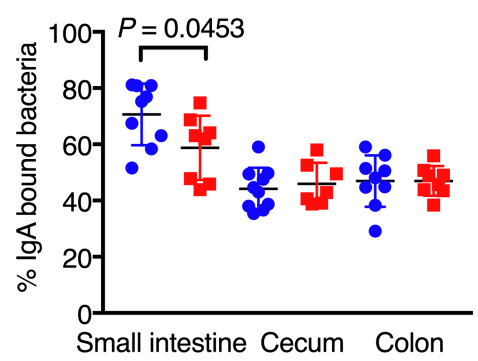

$\mathbf{F}$

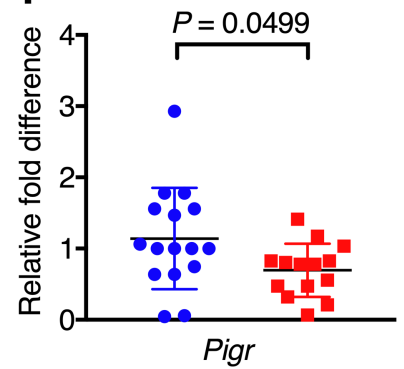

G

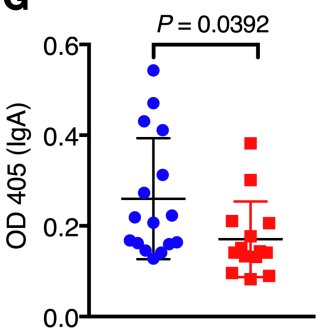

H

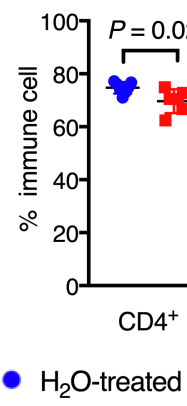

I

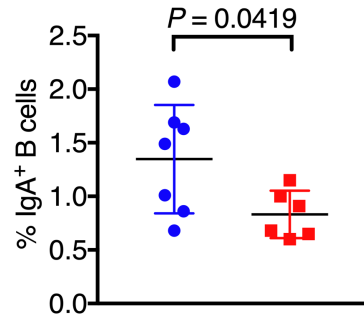

Figure 5. Long-term effect of acetate treatment on IgA response in SPF NOD mice. (A) Timeline of SPF NOD mice gavaged with acetate or water. (B) Longitudinal proportion of IgA-bound stool bacteria before and after gavage $(n=8-9)$. (C) Gut permeability $(n=7-9)$. (D) IgA concentrations in intestinal flush $(n=6-7)$. (E) Percentage of IgA-bound bacteria in the intestinal flush $(n=8-9)$. (F) Intestinal Pigr expression post-acetate treatment ( $n=14-16)$. (G) IgA reactive to gut bacterial products. Stool microbiota from Rag-deficient mice (without any type/form of antibodies) were used to assess IgAs reacting to gut bacterial products. IgAs reacting to the bacterial products in small intestinal flush from the mice with or without acetate treatment were determined by anti-mouse IgA by ELISA $(n=14-16)$. (H) Proportion of CD4+ or CD8 ${ }^{+}$T cells and CD19+ B cells in Peyer's patch $(n=6-7)$. (I) Proportion of splenic IgA+ B cells $(n=6-7)$. Data were pooled from 2 or more independent experiments, and analyzed using either a 2-way ANOVA (B) or a 2-tailed Student's $t$ test (C-I, Data are presented as mean \pm SEM). SPF, specific pathogen-free.

patients also had higher proportions of IgA-bound gut bacteria but lower levels of "free" IgA in the intestine compared with mice colonized with gut bacteria from healthy control subjects; and (f) acetate modulated B cell IgA responses in vitro and in vivo. Thus, our study provides a link between the IgA immune response and gut microbiota modulated by acetate, in the immunopathogenesis of T1D.

$B$ cells were documented to play different roles in the initiation and progression of T1D. Our previous studies and the studies by other scientists showed that B cell-deficient $\left(\mu \mathrm{MT}^{-/-}\right) \mathrm{NOD}$ mice, which are also deficient in immunoglobulin production, are protected against T1D development, indicating that B cells and the antibodies produced by B cells may facilitate the development of T1D (31-33). Among antibodies produced by B cells, IgA has been demonstrated to "coat" a substantial fraction of intestinal bacteria and is an important component of the gut homeostatic barrier together with mucus and antimicrobial peptides (34). In this study, we found that newly diagnosed patients with T1D had an increased level of IgA-bound gut bacteria, in contrast to healthy control subjects. Colonizing GF NOD mice with gut bacteria from patients with T1D changed the host IgA immune response, including an increased ability of IgA to bind gut microbiota and decreased free gut luminal IgA. Zeng and colleagues showed that gut bacteria promote IgG antibody secretion in the host by releasing microbial products that act as antigens and ligands for B cell receptors and TLRs, respectively (35). Other studies 
A

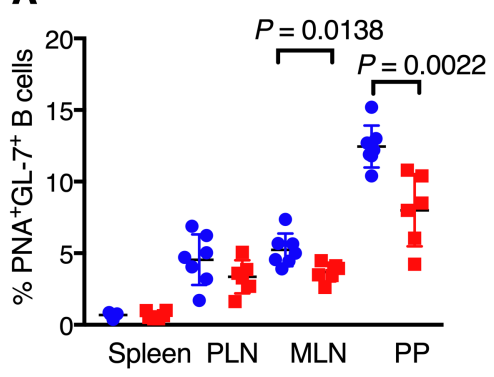

B

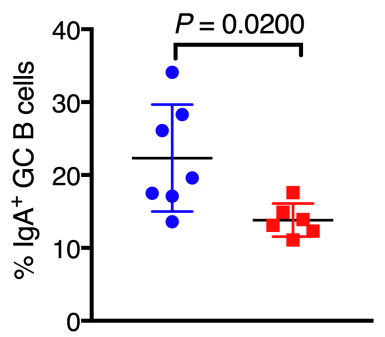

C

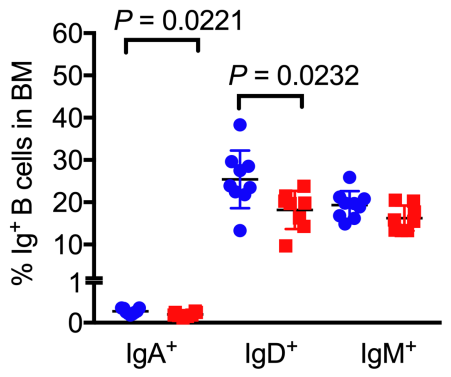

D

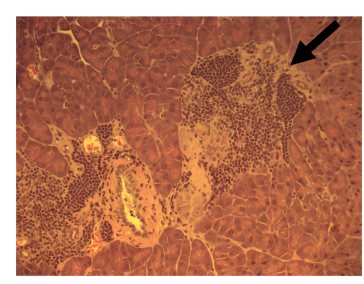

$\mathrm{H}_{2} \mathrm{O}$-treated

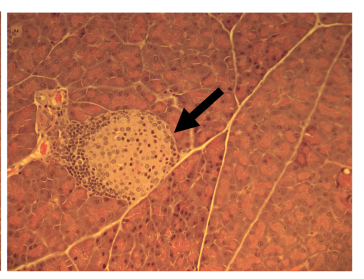

Acetate-treated
E

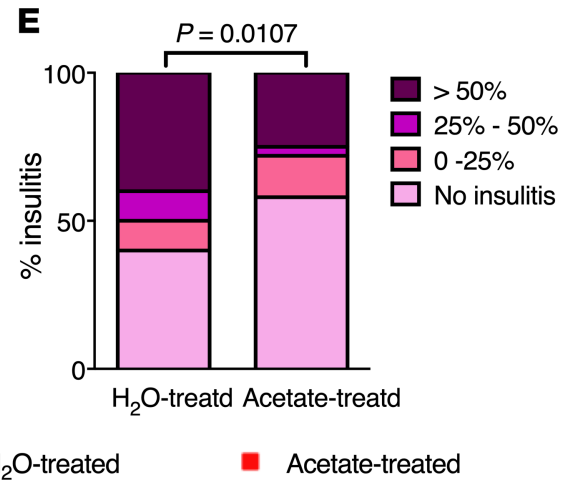

$\mathbf{F}$

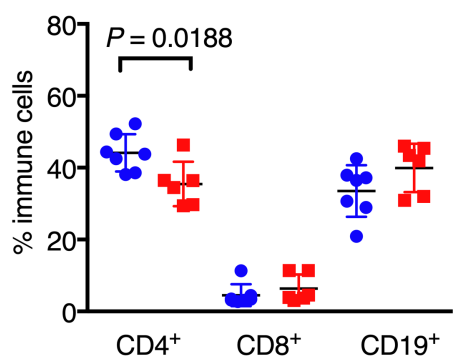

Figure 6. Long-term effect of acetate treatment on IgA response and insulitis in specific pathogen-free NOD mice. (A) Proportion of PNA ${ }^{+} \mathrm{CL}-7^{+}$germinal center B cells $(n=6-7)$. (B) Percentage of splenic IgA+ GC B cells $(n=6-7)$. (C) Proportion of IgA $A^{+}$, IgD', and IgM+ B cells in bone marrow ( $\left.n=8-9\right)$. (D) Representative insulitis images of microscopic views $(\times 200)$ showing differences in immune cell infiltration marked by the black arrows. (E) Summarized percentage of severity of insulitis (total of 143-164 islets were graded from 6-7 mice/group). (F) Infiltrated immune cells in the islets ( $n=6-7)$. Data were pooled from 2 or more independent experiments, and analyzed using either a 2-tailed Student's $t$ test (A-C, and $\mathbf{F}$, Data are presented as mean \pm SEM) or a chi-square test (E). PLN, pancreatic lymph node; MLN, mesenteric lymph node; PP, Peyer's patch.

reported that IgA production can be enhanced by TLR3 agonist poly I:C and TLR5 agonist flagellin $(36,37)$. Further, microbial metabolite SCFAs can either promote or inhibit the production of $\operatorname{IgA}$, depending on the concentration of SCFAs (16). The modulation of IgA production can be mediated by $\mathrm{B}$ cell metabolism and gene expression for plasma cell differentiation and induction of the vitamin A-converting enzyme expression in intestinal epithelial cells $(16,38)$. In this study, we demonstrated that patients with T1D have significantly lower concentrations of stool SCFAs than matched healthy controls; moreover, the levels of SCFAs are associated with an increased level of IgA-bound gut bacteria, suggesting that gut microbiota-derived SCFAs modulate the targeting ability of IgA to specific commensal bacteria in T1D.

Recently, Marino and colleagues reported that feeding NOD mice with combined acetate- and butyrate-enriched diets led to T1D protection, by the reduction of autoreactive T cells and expansion of regulatory T cells (17). The authors also found that gut microbiota such as Christensenella negatively correlated with fecal acetate in NOD mice (17). Interestingly, we found that GF NOD mice colonized with fecal bacteria from patients with T1D have a significantly lower abundance of Christensenella compared with mice colonized with fecal bacteria from healthy control subjects. Moreover, by colonizing GF NOD mice with patients' bacteria, followed by administration of acetate to the mice, we demonstrated that acetate modulated the IgA immune response induced by gut bacteria from the patients with T1D. We also show that acetate ameliorated the self-destruction of islet $\beta$ cells by reducing CD4 ${ }^{+} \mathrm{T}$ cell infiltration in the islets and ameliorated the progression of insulitis in NOD mice. Supporting the findings by Marino and colleagues (17), we found that the long-term treatment with acetate in NOD mice induces Treg cell expansion, but only in PP. Further studies are required to probe the role of Treg cells in IgA immunity in T1D. However, our study revealed that acetate reduced the population of GC B cells in the gut-associated lymphoid tissues and inhibited the molecules essential for B cell differentiation. Although Aid, which is essential for IgA class switching (39), remained unchanged in B cells, Pst transcription factors, such as Pst $\alpha$ and Pst2b, and Irf4 were inhibited in NOD mice that received acetate treatment. Class switching in 
A

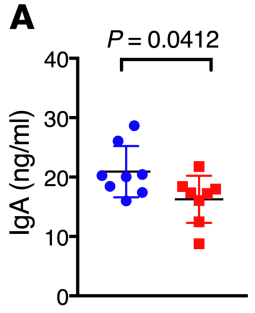

B

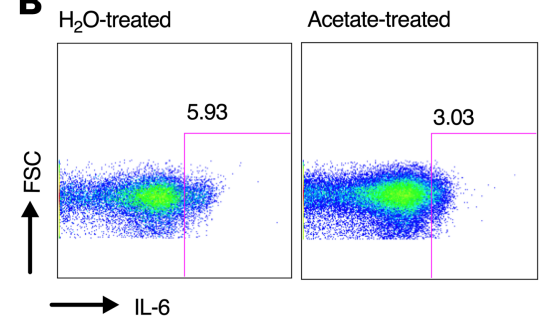

C

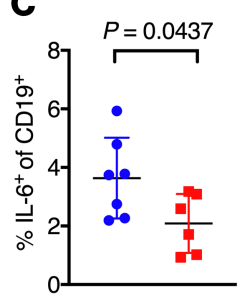

D

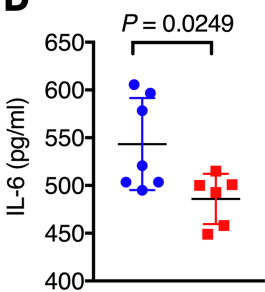

E

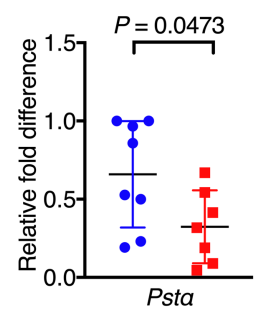

$\mathbf{F}$

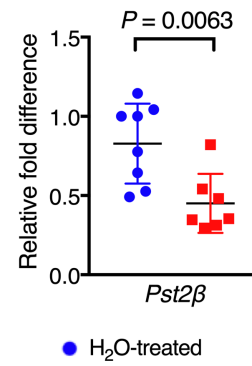

G

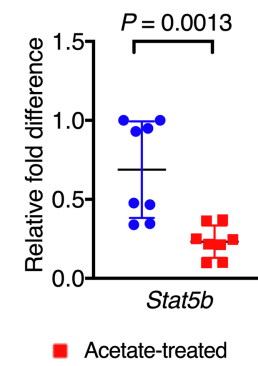

H

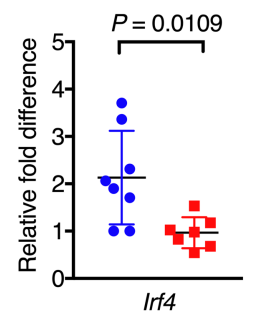

Figure 7. Direct effect of acetate on B cells. Ex vivo splenic B cells were purified from specific pathogen-free NOD mice gavaged with acetate or water for 10 to 12 weeks. The purified $B$ cells were stimulated in vitro in the presence of $10 \mathrm{Mm}$ acetate with $20 \mu \mathrm{g} / \mathrm{mL}$ anti-CD40 mAb and $10 \mu \mathrm{g} / \mathrm{mL}$ LPS for 5 days. (A) IgA concentration in the culture supernatant of stimulated B cells was measured by ELISA $(n=8-9)$. (B) Representative flow cytometric plots of intracellular IL-6 expression of B cells after acetate stimulation. (C) Summary of IL-6-expressing B cells. (D) Secreted IL-6, determined by ELISA, from the culture supernatant of B cells stimulated with acetate $(n=6-7)$. (E-H) Gene expression of B cells, after acetate stimulation, was assessed by qPCR: Pst $\alpha$ (E), Pst2 $\beta$ (F), Stat5b (G), and Irf4 (H). The expression levels were determined using the $2^{-\Delta \Delta C t}$ method by normalizing the housekeeping gene Gapdh. Data combined from 2 independent experiments are presented as mean \pm SEM and were analyzed using a 2-tailed Student's $t$ test (A and $\mathbf{C}-\mathbf{H})$.

$\mathrm{B}$ cells to the IgA isotype requires induction of transcription in the $\mathrm{C} \alpha$ switch region (S $\alpha$ ), which can be initiated by a number of factors including TGF- $\beta 1$, IL-4, IL-6, IL-10, and retinoic acid (34). Our results showed that SCFAs inhibited splenic IL-6 expression in B cells, indicating that reduced IL-6 expression may contribute to the decreased IgA production.

It is known that $\operatorname{IgA}^{+} \mathrm{B}$ cells reside in different lymphoid and nonlymphoid tissues $(23,40-43)$. In this study, we found that acetate treatment inhibited the production of bone marrow $\operatorname{IgA}^{+} \mathrm{B}$ cells in both colonized GF NOD mice and SPF NOD mice. In addition, acetate inhibits the population of GC $\mathrm{B}$ cells in the MLN and PP, which are considered as important primary sites for IgA immune response $(40,44)$. Polymeric Ig receptor (pIgR) is a transmembrane protein expressed on the surface of intestinal epithelial cells and regulates a key pathway for IgA secretion by acting as a transport receptor for IgA (45). After transportation, pIgR becomes a part of the secreted IgA molecule and plays an important role in making the IgA molecule less susceptible to proteolytic digestion, thus increasing the capacity of the IgA molecule to target potential pathogens, thereby inhibiting their attachment to the epithelial surface $(46,47)$. Here we found that acetate suppressed the gene expression of Pigr in the gut, reduced the percentage of IgA-bound bacteria, and decreased the "coating" ability of the free IgA to gut microbiota. Thus, our results reveal that acetate modulates the IgA immune response to commensal bacteria, potentially by decreasing the expression of Pigr, leading to suppressed IgA immunity. Further proof could be provided by testing genetically modified NOD mice with Pigr deficiency and/or overexpression, which could be a future direction.

Taken together, we demonstrate that gut bacteria from patients with T1D alter the host intestinal and systemic IgA immune responses, which are mediated by gut microbiota-derived SCFAs. The contribution of the host intestinal and systemic IgA immune responses, associated with gut microbiota, to directly causing T1D development in humans would be confirmed in a future longitudinal study in prediabetic individuals. However, our cross-sectional study provides novel insights to our knowledge regarding the function of gut microbiota and their metabolites in the immunopathogenesis of T1D. 
A

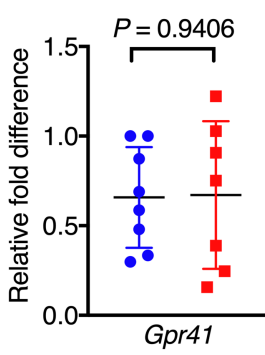

B

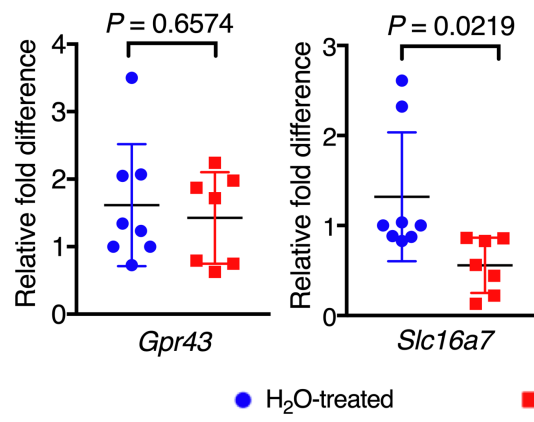

D

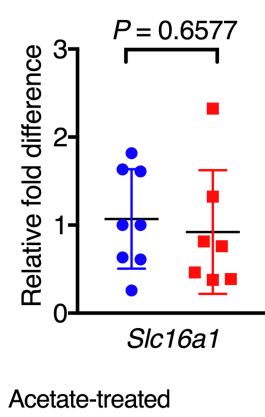

E

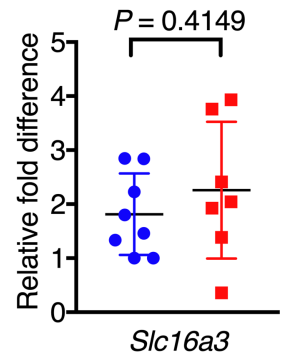

Figure 8. Gene expression of gprs and solute carrier family 16 on B cells. Ex vivo splenic B cells were purified from specific pathogen-free NOD mice gavaged with $200 \mu \mathrm{L}$ of water or equivalent volume of $100 \mathrm{Mm}$ acetate for 10 to 12 weeks, and were stimulated in vitro with $20 \mu \mathrm{g} / \mathrm{mL}$ anti-CD40 $\mathrm{mAb}$ and $10 \mu \mathrm{g} / \mathrm{mL}$ LPS in the presence of $10 \mathrm{Mm}$ acetate for 5 days. Gene expression of acetate-stimulated B cells was assessed by qPCR: Gpr41 (A), Gpr43 (B), Slc16a7 (C), S/c16a1 (D), and SIc16a3 (E). All expression data were determined using the $2^{-\Delta \Delta C t}$ method by normalization with the housekeeping gene $\mathrm{CAPDH}$. Data combined from 2 independent experiments are presented as mean \pm SEM and were assessed for statistical significance using a 2-tailed Student's $t$ test $(n=7-8)$

\section{Methods}

Human samples. Human samples were collected from pediatric patients with T1D, diagnosed within one year of diagnosis, and sex- and age-matched healthy control subjects, aged 6-18 years at the time, in the Diabetes Center of the Second Xiangya Hospital of Central South University, China. Exclusion criteria included: (a) active infection and/or antibiotic treatment, or any infection or antibiotic treatment within the 3 months before the sample collection; (b) active or recent consumption of probiotics; (c) any signs of acute or chronic inflammation; (d) oral contraceptives or pregnancy; (d) any medication, other than for diabetes management (insulin usage); (e) individuals with a known history of immune dysfunction, such as allergy and asthma or other autoimmune diseases including systemic lupus erythematosus, Crohn's disease, rheumatoid arthritis, and multiple sclerosis; (f) individuals with a known history of malignancy or other diseases including gastrointestinal tract, liver, and kidney disorders; and (g) individuals diagnosed with psychiatric disorders including any individuals prescribed psychiatric medications. T1D was diagnosed in accordance with the criteria of the American Diabetes Association (2018). Patients with T1D were selected randomly (with sufficient quantity of the study materials) and control subjects were age and sex matched.

Mice. Mice used in this study were housed in strict GF or SPF facilities with a 12-hour-dark/light cycle at Yale University. The GF NOD breeders were provided by A. Chervonsky (University of Chicago, Illinois, USA). SPF NOD mice and $\mathrm{Rag}^{-1-}$ NOD mice were originally obtained from The Jackson Laboratory and were maintained at Yale University. At least 6 or more mice per experiment were used for the purpose of reproducibility and statistical significance. Mice were randomly allocated in the different experimental groups from different cages, ensuring that mice of the same age and sex were taken from a mixed pool of different breeders. All data from mouse studies were representative of 2 or more independent experiments.

Extraction of gut bacterial DNA. Fresh human stool samples, collected using sterile procedure, were immediately aliquoted and frozen at $-80^{\circ} \mathrm{C}$ before use. Stool samples from humans or mice were resuspended in TE buffer (10 Mm Tris and $1 \mathrm{Mm}$ EDTA, $\mathrm{pH}=8$ ) containing $0.5 \%$ SDS and $200 \mu \mathrm{g} / \mathrm{mL}$ Proteinase $\mathrm{K}$. After incubation at $37^{\circ} \mathrm{C}$ for 1 hour, the samples were homogenized in solution containing phenol/chloroform/isoamyl alcohol, 20\% SDS, and zirconium silica beads. Following centrifugation (15 minutes, $4^{\circ} \mathrm{C}, 12,000 \mathrm{~g}$ ), the upper aqueous layer containing DNA was removed and transferred to a new tube. Bacterial DNA was subsequently precipitated by isopropanol and washed with $70 \%$ ethanol.

$16 S$ rRNA sequencing and data analysis. Fresh samples were collected from both outpatients and inpatients using a sterile wooden spatula into a sterile container. The collected stool samples were immediately aliquoted into small vials in the laboratory and frozen at $-80^{\circ} \mathrm{C}$ within 2 hours from the time of sample collection. Bacterial 16S rRNA V4 region was amplified from each DNA sample by PCR using barcoded primer pairs (5'-GTGCCAGCMGCCGCGGTAA-3') and (5'-GGACTACHVGGGTWTCTAAT-3'). The PCR products were purified using a QIAGEN gel extraction kit. After the quantification of DNA concentration with NanoDrop II, samples were pooled for pyrosequencing using the Ion Torrent Personal Genome Machine sequencing system (Life Technologies). The sequencing results were analyzed with the 
QIIME software package and UPARSE pipeline to pick operational taxonomic units (OTUs). $\alpha$ Diversity was measured by observed species number, phylogenetic diversity, or Chao richness in the bacterial communities. $\beta$ Diversity was calculated to compare differences between microbial communities, shown as principal coordinate analysis. Taxonomy assignment was performed at various levels using representative sequences of each OTU. The sequencing data have been deposited in the SRA database (accession PRJNA615687, T1D patient and healthy control stool samples; PRJNA615637, fecal samples of GF NOD mice colonized with human bacteria; and PRJNA615380, fecal samples of GF NOD mice colonized with T1D patient bacteria followed with acetate treatment).

IgA-bound bacterial staining. Human or mouse fecal samples were resuspended at $1 \mathrm{~g} / \mathrm{mL}$ in sterile PBS and homogenized by vortexing vigorously for 30 seconds. Intestinal lumen contents were collected from GF NOD mice or SPF NOD mice by flushing the small intestine, cecum, and colon with $10 \mathrm{~mL}$ of sterile PBS. All the samples were centrifuged ( $300 \mathrm{~g}, 1$ minute, room temperature) to remove the large debris and further spun at $12,000 \mathrm{~g}$ for 5 minutes to pellet bacteria. Supernatant was frozen at $-20^{\circ} \mathrm{C}$ for free $\operatorname{IgA}$ analysis. Bacterial pellets were washed and resuspended in $50 \mu 1$ of $1 \%$ BSA/PBS for 15 minutes at room temperature before the incubation with anti-IgA antibody for 30 minutes at room temperature. Samples were then washed and resuspended in sterile PBS, for analysis on a BD Biosciences LSRII Flow Cytometer, and further analyzed using FlowJo software. Bacteria from human stool were stained with anti-human IgA-PE antibody (Miltenyi). Bacteria from mice were stained with goat anti-mouse IgA-PE (Southern Biotech) and isotype control antibodies (BioLegend).

Oral gavage. Fresh human stool samples were aliquoted immediately after collection. Bacterial pellets from fresh stool samples were resuspended in sterile PBS and stored in $50 \%$ glycerol at $-80^{\circ} \mathrm{C}$. Before gavage, frozen bacteria were quickly thawed in $37^{\circ} \mathrm{C}$ water bath and then washed twice in sterile PBS. Bacterial colony-forming units (CFUs) were determined by measurement of optical density (OD) with a pretitrated control bacterium E. coli. 4-week-old GF NOD mice were colonized with $100 \mu \mathrm{L}$ sterile PBS containing $2 \times 10^{8} \mathrm{CFUs}$ of stool bacteria from either individuals with T1D or controls. Colonized mice were terminated 1 week after gavage. For the in vivo acetate experiments, 4-week-old GF NOD mice were colonized with $100 \mu \mathrm{L}$ sterile PBS containing $2 \times 10^{8} \mathrm{CFUs}$ stool bacteria from individuals with T1D. One week after colonization, the mice were orally gavaged with $100 \mathrm{Mm}$ sodium acetate in $200 \mu \mathrm{L}$ of sterile water $\left(\mathrm{H}_{2} \mathrm{O}\right)$ every 2 days. These mice were terminated 2 weeks after colonization (one-week after acetate or water treatment). For SPF NOD mice, 4-week-old female mice were gavaged with $100 \mathrm{Mm}$ sodium acetate in $200 \mu \mathrm{L}$ of sterile water every 2 days over 10 to 12 weeks. Control mice in each experiment were gavaged with $200 \mu \mathrm{L}$ of sterile water.

Determination of bacterial product-reactive $\operatorname{Ig} A$. Fecal microbiota $\left(1 \times 10^{9} \mathrm{CFU} / \mathrm{mL}\right)$ from 2-month-old $\mathrm{Rag}^{-1-} \mathrm{NOD}$ mice were alkaline lysed by resuspending in $0.1 \mathrm{M} \mathrm{NaHCO}_{3}$ buffer ( $\mathrm{pH}$ 9.1) and debris-free phase was used to coat the wells of a 96-well ELISA plate $\left(50 \mu \mathrm{L} /\right.$ well) overnight at $4^{\circ} \mathrm{C}$. After washing, plates were blocked with $1 \%$ BSA/PBS for 1 hour at $37^{\circ} \mathrm{C}$. Serum or gut flushes (nonbacteria-containing supernatants of small intestine, cecum, and large intestine) from SPF NOD females treated with water or acetate were assessed. Serum was diluted 1:100 in 1\% BSA/PBS, whereas gut flushes were used without further dilution. Samples (all in duplicate) were added to the appropriate wells and the plates were incubated at $37^{\circ} \mathrm{C}$ for 1.5 hours. Samples were washed and then incubated with IgA-AP (1:1000 dilution; Southern-Biotech) at $37^{\circ} \mathrm{C}$ for a further 1.5 hours. Following the final wash, substrate PNPP was added. The reaction was stopped by adding $1 \mathrm{~N} \mathrm{NaOH}$. Samples were then analyzed on a microplate spectrophotometer (PerkinElmer) at $405 \mathrm{~nm}$ (OD). Antibody concentrations were determined by linear regression.

Lamina propria immune cell isolation. Mouse large intestine was harvested. Luminal contents were flushed with $10 \mathrm{~mL}$ sterile PBS. After removing PP, the intestine was cut longitudinally into 0.5 -cm lengths and washed in PBS. Gut tissue was then shaken on a shaking incubator for 20 minutes at $250 \mathrm{rpm}$ at $37^{\circ} \mathrm{C}$ in prewarmed HBSS containing $1 \mathrm{Mm}$ EDTA. Tissue was vortexed at maximum speed for 30 seconds. The solution was then filtered through nylon mesh. The remaining gut tissue was further digested with $1 \mathrm{mg} /$ $\mathrm{mL}$ collagenase D and 500 U DNAse1 in RPMI medium and incubated on a shaking incubator for 1 hour with rotation at $250 \mathrm{rpm}, 37^{\circ} \mathrm{C}$ for lamina propria (LP) lymphocyte isolation. Immune cells from LP were purified by Percoll (MilliporeSigma) gradient.

Gut permeability assay. Mice were fasted overnight for 13 hours before the gavage with FITC-dextran (600 mg/kg) (MilliporeSigma). Baseline blood samples were collected from the mice before oral gavage with FITC-dextran in sterile PBS. Two hours after gavage, food supply was restored to the mice. Another 
2 hours later, blood samples were collected from the mice. Blood was centrifuged $(2300 \mathrm{~g}, 5$ minutes, room temperature) to isolate serum. Serum samples were diluted 1:1 in PBS in a 96-well plate and serum FITC-dextran concentration was determined using a fluorescence spectrophotometer (PerkinElmer). Serum samples from non-FITC-dextran gavaged NOD mice were used as baseline. Standard curves were generated using known concentrations of FITC-dextran diluted in control serum. Concentrations were determined using linear regression.

$B$ cell purification and cell culture. Splenocytes were isolated from 14- to 16-week-old acetate- or water-gavaged female SPF NOD mice. Splenic B cells were purified following the MagniSort Mouse B cell Enrichment Kit (eBioscience) with a purity of $93 \%$ to $97 \%$ verified by flow cytometry. Purified B cells were stimulated with $20 \mu \mathrm{g} / \mathrm{mL}$ anti-CD40 mAb (FGK4.5, BioXcell) and $10 \mu \mathrm{g} / \mathrm{mL}$ LPS (MilliporeSigma) with $10 \mathrm{Mm}$ acetate or sterile PBS (solvent for acetate) for 5 days. Cell culture supernatants were collected for secreted IgA and cytokine measurement at the end of the culture.

Monoclonal antibody staining of cells for analysis by flow cytometry. One million $\left(1 \times 10^{6}\right)$ cells from different mouse lymphoid tissues were incubated with an Fc blocker in PBS at room temperature for 20 minutes before cell surface staining. For intracellular cytokine staining, cells were incubated at $37^{\circ} \mathrm{C}$ for 4 hours in the presence of $50 \mathrm{ng} / \mathrm{mL}$ PMA (MilliporeSigma), $500 \mathrm{ng} / \mathrm{mL}$ of ionomycin (MilliporeSigma), and $1 \mu 1$ of Golgi plug (BD Biosciences), followed by mAbs to surface molecules and intracellular cytokine staining. Samples were analyzed on a BD LSRFortessa Flow Cytometer and results were analyzed by FlowJo 8.8.6 (Tree star).

Histopathology and insulitis score. Pancreata from 14- to 16-week-old SPF NOD (orally gavaged with acetate or water) were fixed in $10 \%$ buffered formalin and paraffin-embedded. Tissues were stained with $\mathrm{H} \& \mathrm{E}$ and insulitis was scored by light microscopy by an individual blinded to the study. The following insulitis grading was used: 0 , no infiltration; I, $<25 \%$ infiltration; II, $25 \%-50 \%$ infiltration; III, $>50 \%$ infiltration. 143-164 islets were scored for insulitis in each group ( $n=6-7$ mice).

Murine antibody ELISA. Murine IgA levels were measured by direct ELISA (Southern Biotech) in the samples of serum, culture supernatants and luminal gut contents from colonized GF NOD mice and SPF NOD mice. Briefly, wells of a 96-well plate were coated with samples or standards. After washing and blocking (1 hour, room temperature with 1\% BSA in PBS), the plates were then incubated with AP-conjugated goat anti-mouse IgA (2 hours, room temperature). Samples were subsequently washed and substrate PNPP (MilliporeSigma) was added. The reaction was stopped by adding $1 \mathrm{M} \mathrm{NaOH}$. Samples were analyzed on a microplate spectrophotometer (PerkinElmer) at $405 \mathrm{~nm}$ (OD). Antibody concentrations were determined by linear regression.

Cytokine ELISA. Murine IL-6 was measured using the Mouse IL-6 ELISA kit following the manufacturer's instructions (BioLegend).

Real-time qPCR. RNA from purified B cells or small intestinal tissue was extracted using Trizol reagent and RNeasy Mini Plus Kit (QIAGEN). After quantification, $1 \mu \mathrm{g}$ RNA was used for cDNA synthesis using the iScript cDNA Synthesis Kit (Invitrogen). Samples were analyzed on an iCycler qPCR machine (Bio$\mathrm{Rad})$. Gene expression was determined using the $2^{-\triangle \Delta C t}$ method and normalization with the housekeeping gene, GAPDH. Primers sequences are listed in Supplemental Table 2. Each sample was assayed in duplicate and the experiments were repeated at least twice.

Antibodies and reagents. The fluorochrome-conjugated $\mathrm{mAbs}$ used in this study include: anti-human PE-IgA (Miltenyi, catalog 130-093-128); anti-mouse PE-IgA (0.2 mg/mL, Southern Biotech, catalog 116428); anti-mouse PE-Cy7-TCR $\beta$ (0.2 mg/mL, BioLegend, catalog 109222); anti-mouse PE-Foxp3 (0.2 $\mathrm{mg} / \mathrm{mL}$, eBioscience, catalog 2001196); anti-mouse PE-IL-6 (0.2 mg/mL, BioLegend, catalog 504504); anti-mouse PE-Cy7-CD8 (0.2 mg/mL, BioLegend, catalog 100722); anti-mouse APC-IgM (0.2 mg/mL, BioLegend, catalog 406509); anti-mouse Alexa Fluor 147-GL-7 (0.5 mg/mL, BioLegend, catalog 144606); anti-mouse FITC-CD21 (0.5 mg/mL, BioLegend, catalog 123408); anti-mouse FITC-CD4 (0.5 mg/mL, BioLegend, catalog 100406); anti-mouse FITC-TNF- $\alpha$ (0.5 mg/mL, BioLegend, catalog 506304); antimouse PerCp-Cy5.5-CD45 (0.2 mg/mL, BioLegend, catalog 102132); anti-mouse PerCp-Cy5.5-CD25 (0.2 $\mathrm{mg} / \mathrm{mL}$, BioLegend, catalog 101912); anti-mouse PerCp-Cy5.5-IFN- $\gamma(0.2 \mathrm{mg} / \mathrm{mL}$, BioLegend, catalog 101912); anti-mouse APC/Cy7-CD19 (0.2 mg/mL, BioLegend, catalog 115530); anti-mouse APC/Cy7CD4 (0.2 mg/mL, BioLegend, catalog 100414); anti-mouse APC/Cy7-IgD (0.2 mg/mL, BioLegend, catalog 108406, RB6-8C5); anti-mouse Pacific Blue-CD23 (0.5 mg/mL, BioLegend, catalog 101616); and anti-mouse Pacific Blue-CD4 (0.5 mg/mL, BioLegend, catalog 100428). Bacterial DNA isolation and pyrosequencing reagents were purchased from Qiagen and Roche, respectively. 
Statistics. Statistical analysis was performed using GraphPad Prism software Vs7 for Mac. Data are expressed as mean \pm SEM or median (25th-75th percentile). Differences between groups were analyzed using a 2-tailed Student's $t$ test, 1-way ANOVA or 2-way ANOVA. Correlations were analyzed using a 2 -tailed Pearson correlation coefficient test and/or linear regression. Statistical analysis of microbial $\beta$ diversity was conducted using an analysis of similarities. Differences between microbial species were determined following analysis using multiple $t$ tests with Bonferroni's correction. $P$ value and statistical analysis for each experiment are reported in the figure legends. A $P$ value of less than 0.05 was considered significant.

Study approval. The use of animals in this study was approved by the Institutional Animal Care and Use Committee at Yale University. For human studies, all participants or their guardians provided informed consent. The research was approved by the Ethics Committee of the Second Xiangya Hospital. The study was conducted according to Declaration of Helsinki principles.

\section{Author contributions}

LW conceived the study. LW, ZZ, JH, and JAP designed the study. JH and JAP conducted the experiments, analyzed data and wrote the manuscript. JH and JAP are co-first authors. JH conducted both clinical and bench experiments and is, therefore, listed in the first place. JP, YH, SS, Y. Xing, GH, XL, FH, ZX, Y. Xiao, SL, and CC conducted some of the studies and analyzed the results. LW and FSW revised the manuscript. All authors have agreed the contents and approved manuscript submission.

\section{Acknowledgments}

We thank all the subjects who participated in the study, their parents for their support, and all the nurses and technicians who helped us collect the samples. We thank Weisan Chen for his valuable comments on the manuscript. This work was supported by the Molecular Genetic \& Diabetes Mouse Core of Yale Diabetes Center (DK 045735 and HD 097808, to LW); a JDRF Postdoctoral Research Fellowship (3-PDF-2016197-A-N, to JAP); the National Key R\&D Program of China (2016YFC1305000 and 2016YFC1305001, to ZZ); the National Natural Science Foundation of China (81820108007 and 8181001262, to ZZ); and the Science and Technology Major Project of Hunan Province (2017SK1020, to ZZ).

Address correspondence to: Li Wen, S141, TAC 300, Cedar Street, New Haven, Connecticut 06520-8103, USA. Phone: 203.785.7186; Email: li.wen@yale.edu. Or to: Zhiguang Zhou, 139 Renmin Road, Changsha, Hunan 410011, China. Phone: 86.731.85292154; Email: zhouzhiguang@csu.edu.cn.

1. Patterson CC, et al. Trends in childhood type 1 diabetes incidence in Europe during 1989-2008: evidence of non-uniformity over time in rates of increase. Diabetologia. 2012;55(8):2142-2147.

2. Barnett AH, Eff C, Leslie RD, Pyke DA. Diabetes in identical twins. A study of 200 pairs. Diabetologia. 1981;20(2):87-93.

3. Redondo MJ, et al. Heterogeneity of type I diabetes: analysis of monozygotic twins in Great Britain and the United States. Diabetologia. 2001;44(3):354-362.

4. Vehik K, et al. Trends in high-risk HLA susceptibility genes among Colorado youth with type 1 diabetes. Diabetes Care. 2008;31(7):1392-1396.

5. Wen L, et al. Innate immunity and intestinal microbiota in the development of Type 1 diabetes. Nature. 2008;455(7216):1109-1113.

6. Giongo A, et al. Toward defining the autoimmune microbiome for type 1 diabetes. ISME J. 2011;5(1):82-91.

7. de Goffau MC, et al. Fecal microbiota composition differs between children with $\beta$-cell autoimmunity and those without. Diabetes. 2013;62(4):1238-1244.

8. Brown K, et al. Prolonged antibiotic treatment induces a diabetogenic intestinal microbiome that accelerates diabetes in NOD mice. ISME J. 2016;10(2):321-332.

9. Hu Y, Peng J, Li F, Wong FS, Wen L. Evaluation of different mucosal microbiota leads to gut microbiota-based prediction of type 1 diabetes in NOD mice. Sci Rep. 2018;8(1):15451.

10. Kostic AD, et al. The dynamics of the human infant gut microbiome in development and in progression toward type 1 diabetes. Cell Host Microbe. 2015;17(2):260-273.

11. Vatanen T, et al. Variation in microbiome LPS immunogenicity contributes to autoimmunity in humans. Cell. 2016;165(4):842-853.

12. Tai N, et al. Microbial antigen mimics activate diabetogenic CD8 T cells in NOD mice. J Exp Med. 2016;213(10):2129-2146.

13. Cole DK, et al. Hotspot autoimmune T cell receptor binding underlies pathogen and insulin peptide cross-reactivity. J Clin Invest. 2016;126(9):3626.

14. Hebbandi Nanjundappa R, et al. A gut microbial mimic that hijacks diabetogenic autoreactivity to suppress colitis. Cell. 2017;171(3):655-667.e17.

15. Smith PM, et al. The microbial metabolites, short-chain fatty acids, regulate colonic Treg cell homeostasis. Science. 2013;341(6145):569-573. 
16. Kim M, Qie Y, Park J, Kim CH. Gut microbial metabolites fuel host antibody responses. Cell Host Microbe. 2016;20(2):202-214.

17. Mariño E, et al. Gut microbial metabolites limit the frequency of autoimmune T cells and protect against type 1 diabetes. Nat Immunol. 2017;18(5):552-562.

18. Vatanen T, et al. The human gut microbiome in early-onset type 1 diabetes from the TEDDY study. Nature. 2018;562(7728):589-594.

19. de Groot PF, et al. Distinct fecal and oral microbiota composition in human type 1 diabetes, an observational study. PLoS One. 2017;12(12):e0188475.

20. Conley ME, Delacroix DL. Intravascular and mucosal immunoglobulin A: two separate but related systems of immune defense? Ann Intern Med. 1987;106(6):892-899.

21. Macpherson AJ, Uhr T. Induction of protective IgA by intestinal dendritic cells carrying commensal bacteria. Science. 2004;303(5664):1662-1665.

22. Grootjans J, et al. Epithelial endoplasmic reticulum stress orchestrates a protective IgA response. Science. 2019;363(6430):993-998.

23. Bunker JJ, et al. Natural polyreactive IgA antibodies coat the intestinal microbiota. Science. 2017;358(6361):eaan6619.

24. Palm NW, et al. Immunoglobulin A coating identifies colitogenic bacteria in inflammatory bowel disease. Cell. 2014;158(5):1000-1010.

25. Paun A, et al. Association of HLA-dependent islet autoimmunity with systemic antibody responses to intestinal commensal bacteria in children. Sci Immunol. 2019;4(32):eaau8125.

26. Rojas OL, et al. Recirculating intestinal IgA-producing cells regulate neuroinflammation via IL-10. Cell. 2019;176(3):610-624.e18.

27. Roldán E, Brieva JA. Terminal differentiation of human bone marrow cells capable of spontaneous and high-rate immunoglobulin secretion: role of bone marrow stromal cells and interleukin 6. Eur J Immunol. 1991;21(11):2671-2677.

28. Cassese G, et al. Plasma cell survival is mediated by synergistic effects of cytokines and adhesion-dependent signals. J Immunol. 2003;171(4):1684-1690.

29. Brown AJ, et al. The Orphan G protein-coupled receptors GPR41 and GPR43 are activated by propionate and other short chain carboxylic acids. J Biol Chem. 2003;278(13):11312-11319.

30. Morris ME, Felmlee MA. Overview of the proton-coupled MCT (SLC16A) family of transporters: characterization, function and role in the transport of the drug of abuse $\gamma$-hydroxybutyric acid. AAPS J. 2008;10(2):311-321.

31. Wong FS, Visintin I, Wen L, Granata J, Flavell R, Janeway CA. The role of lymphocyte subsets in accelerated diabetes in nonobese diabetic-rat insulin promoter-B7-1 (NOD-RIP-B7-1) mice. J Exp Med. 1998;187(12):1985-1993.

32. Serreze DV, et al. B lymphocytes are essential for the initiation of T cell-mediated autoimmune diabetes: analysis of a new "speed congenic" stock of NOD.Ig mu null mice. J Exp Med. 1996;184(5):2049-2053.

33. Akashi T, et al. Direct evidence for the contribution of B cells to the progression of insulitis and the development of diabetes in non-obese diabetic mice. Int Immunol. 1997;9(8):1159-1164.

34. Bunker JJ, Bendelac A. IgA responses to microbiota. Immunity. 2018;49(2):211-224.

35. Zeng MY, et al. Gut microbiota-induced immunoglobulin G controls systemic infection by symbiotic bacteria and pathogens. Immunity. 2016;44(3):647-658.

36. Xu W, et al. Viral double-stranded RNA triggers Ig class switching by activating upper respiratory mucosa B cells through an innate TLR3 pathway involving BAFF. J Immunol. 2008;181(1):276-287.

37. $\mathrm{He} \mathrm{B}$, et al. Intestinal bacteria trigger $\mathrm{T}$ cell-independent immunoglobulin $\mathrm{A}(2)$ class switching by inducing epithelial-cell secretion of the cytokine APRIL. Immunity. 2007;26(6):812-826.

38. Goverse G, et al. Diet-derived short chain fatty acids stimulate intestinal epithelial cells to induce mucosal tolerogenic dendritic cells. J Immunol. 2017;198(5):2172-2181.

39. Muramatsu M, Kinoshita K, Fagarasan S, Yamada S, Shinkai Y, Honjo T. Class switch recombination and hypermutation require activation-induced cytidine deaminase (AID), a potential RNA editing enzyme. Cell. 2000;102(5):553-563.

40. McWilliams M, Phillips-Quagliata JM, Lamm ME. Mesenteric lymph node B lymphoblasts which home to the small intestine are precommitted to IgA synthesis. J Exp Med. 1977;145(4):866-875.

41. Moro-Sibilot L, et al. Mouse and human liver contain immunoglobulin A-secreting cells originating from Peyer's patches and directed against intestinal antigens. Gastroenterology. 2016;151(2):311-323.

42. Roux ME, McWilliams M, Phillips-Quagliata JM, Weisz-Carrington P, Lamm ME. Origin of IgA-secreting plasma cells in the mammary gland. J Exp Med. 1977;146(5):1311-1322.

43. Wilmore JR, et al. Commensal microbes induce serum IgA responses that protect against polymicrobial sepsis. Cell Host Microbe 2018;23(3):302-311.e3.

44. Craig SW, Cebra JJ. Peyer's patches: an enriched source of precursors for IgA-producing immunocytes in the rabbit. J Exp Med. 1971;134(1):188-200.

45. Kaetzel CS. The polymeric immunoglobulin receptor: bridging innate and adaptive immune responses at mucosal surfaces. Immunol Rev. 2005;206:83-99.

46. Johansen FE, Kaetzel CS. Regulation of the polymeric immunoglobulin receptor and IgA transport: new advances in environmental factors that stimulate pIgR expression and its role in mucosal immunity. Mucosal Immunol. 2011;4(6):598-602.

47. Turula H, Wobus CE. The role of the polymeric immunoglobulin receptor and secretory immunoglobulins during mucosal infection and immunity. Viruses. 2018;10(5):E237. 